\title{
Belt Slackness Detection for the Automobile System
}

\author{
C. Sathian, B. Kumar and C. Raju
}

\begin{abstract}
General opinion for the cause of overheating in the engine is scarcity of water in the radiator which causes the engine to seize. Normally refilling the radiators would solve the problem but we have several proven cases where engines still seize due to loosening of belts. Though the current technology allows detection of engine temperature, there is no technique to detect this fault and to alert the driver.

Our paper advocates a feasible solution for the above mentioned problem. A simple circuit with a potentiometer and LM3914 are employed to indicate any deviations in the tension or slackness in the belt. This also helps in preventing other serious consequences that can occur due to improper functioning of the timing belt which is used to drive the cam shaft in an overhead camshaft.
\end{abstract}

Keywords--- Belt Tension, Automobile Engine, LM3914, Potential Divider, Slackness

\section{INTRODUCTION}

$\mathrm{T}$ HIS paper deals with the occurrence of problems that would take place due to slackening of belt, the belt can be either timing belt or drive belt. This paper contains the feasible solutions to handle it. Detecting the slackness of a belt drive in an engine and indicating it to the driver is a preventive measure; if the loosening level is increased then ultimately the functioning of components like water pump, alternator, and A.C components will be in vain.

\section{A. Belts used in Engine \\ i. $\quad$ Serpentine Belt (Drive Belt)}

A serpentine belt is used to drive accessories installed on a car engine, such as an alternator, water pump, power steering pump, air-conditioner, compressor, etc. Sometimes a serpentine belt could also be called a "drive belt" or "accessories belt". Some cars have a single serpentine belt running all the accessories; other cars may have two or more drive belts. [1] To function properly, a serpentine belt needs to be under certain tension. In some older cars a belt tensioner is used which is to be adjusted periodically. If not adjusted in time then, the belt gets loose and starts slipping, making a loud

C. Sathian, Veltech Engineering College, Veltech-Alamathi Road, Avadi, Chennai.E-mail:sathian.chidambaram@gmail.com

B. Kumar, Student Mechanical Engineering, Veltech Engineering College, Veltech-Alamathi Road, Avadi, Chennai. E-mail: iambkumar@gmail.com

C. Raju, Student Mechanical Engineering, Veltech Engineering College, Veltech-Alamathi Road, Avadi, Chennai.E-mail: rajumech09@gmail.com whistling noise. Commonly you would hear this noise at a startup or when turning the steering wheel to the limit [2].

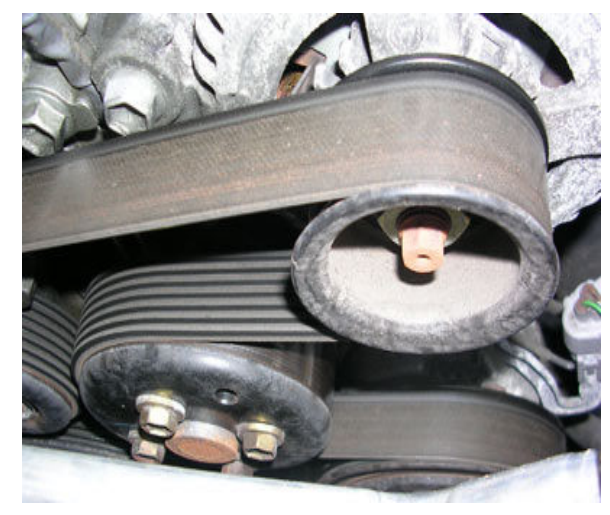

Figure 1: Drive Belt

\section{ii. Timing Belt}

Some genius has said 'life is all about timing', and this even applies to machines. For any vehicle model of any manufacturer, the timing chain or the timing belt is amongst those auto parts, which serve a very important purpose in the vehicle and for a very long time. And any of the timing belt problems need to be addressed sooner than later [3].

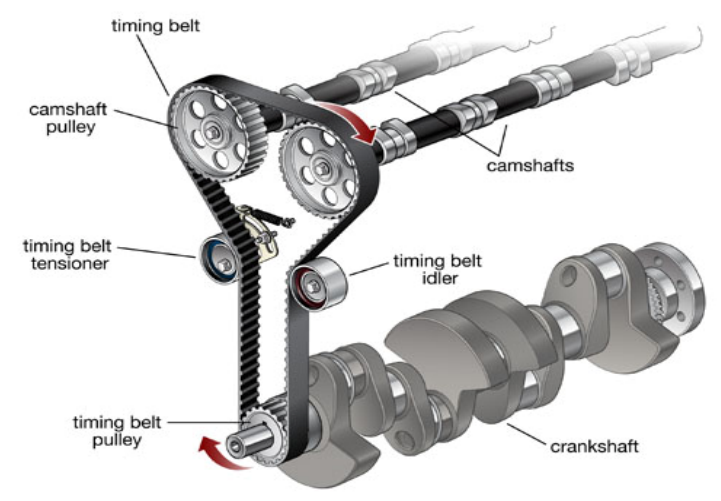

Figure 2: Timing Belt

In an internal combustion engine, a timing belt connects the camshaft to the crankshaft and thus controls the opening and closing action of the intake and exhaust valves in the cylinder. Four strokes engines are designed in such a way that, the intake valves open at the start of the first revolution and close at the end of it. Similarly, the exhaust valve will open at the start of the fourth revolution and closes at the end of it, before the intake valve opens again. This synchronization is entirely controlled by the timing belt and it therefore plays an important role over here, by synchronizing the camshaft with 
the crankshaft. [4]

\section{LITERATURE SURVEY}

Work regarding Monitoring and Positioning of the belt is analyzed by Mr. Dewey D. Henderson in his manuscript titled "Belt Tensioner", (Patent Number: US4596538). It describes the working of a Belt engaging unit to hold the belt. But the drive belt used in an engine cannot utilize the above mentioned tensioner. Andrew L. Bartos provided a manuscript titled "Automatic belt tensioner for vehicle combined startergenerator" According to him a belt tensioner assembly for a vehicle combined starter generator uses shifting reaction torque to provide the tension. Also Klaus, Bytzek in their paper "Timing belt tensioner with damped constant spring tensioning and belt tooth disengagement prevention" (Patent Number: US4583962) describes the prevention of belt tooth disengagement.

\section{LOOSENING OF BELT}

\section{A. Slip}

In belt drive the power is transmitted by the frictional resistance between the belt and pulleys. If the frictional resistance between the driver pulley and belt is insufficient then it may cause some forward motion of the driver pulley without carrying the belt with it. Similarly if the frictional resistance between the belt and driven pulley is insufficient it may cause some forward motion of the belt without carrying the driven pulley with it. In such a case the difference between the speed of driver pulley and belt, belt and driven pulley is called slip and is generally expressed as percentage. [5]

\section{B. Manifestation of Slip}

When the Maruti 800 starts, it initially idles at $700 \mathrm{rpm}$. The pulley ratio between the crankshaft and the alternator is $3: 1$. Thus, the alternator rotates at a minimum of $2100 \mathrm{rpm}$. If the slip increases then the speed fed to the alternator is decreased.

\section{Causes and Consequences of Loosening}

The tension in the belt should remain in a particular level or else the belt will loose and which will lead to slipping of belts.

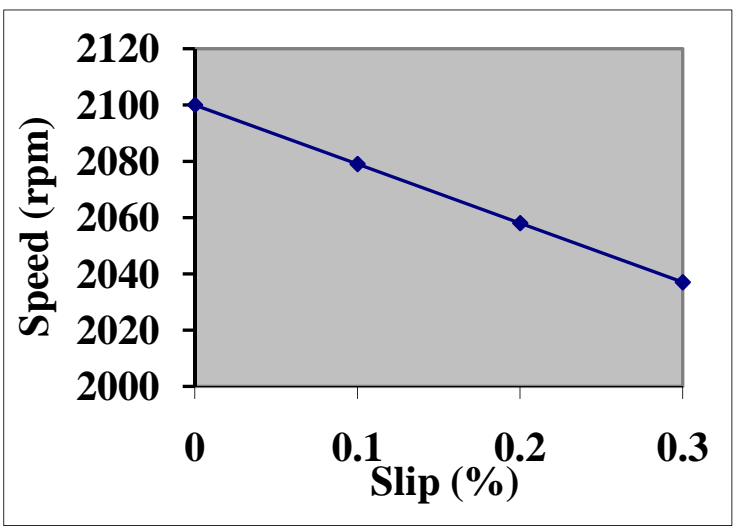

Figure 3: Decay of Speed of Alternator (Maruti 800)
The reasons for loosening of belts are centrifugal force, fatigue load, wear and tear in the belt, or manufacturing defect. Belt drives depend on friction to operate but, if the friction is excessive, there will be waste of energy and rapid wear of the belt. Factors which affect belt friction include belt tension, contact angle and the materials from which the belt and pulleys are made. If the timing belt is not functioning properly then, the consequences are, overheating and leaking in the engine, more Than normal exhaust emission, abrupt change in engine noise, trouble while starting the vehicle

Table 1: Decay of Speed in RPM

\begin{tabular}{|c|c|c|}
\hline $\begin{array}{c}\text { Serial } \\
\text { number }\end{array}$ & SLIP (\%) & $\begin{array}{c}\text { SPEED OF THE } \\
\text { ALTERNATOR(rpm) }\end{array}$ \\
\hline 1 & 0.0 & 2100 \\
\hline 2 & 0.1 & 2079 \\
\hline 3 & 0.2 & 2058 \\
\hline 4 & 0.3 & 2037 \\
\hline
\end{tabular}

D. Malfunction of the following Components

\section{i. Water Pump}

A water pump is an important part of the engine cooling system. It provides circulation of the engine coolant (antifreeze) through the cooling system. A water pump pushes the coolant through the passages (water jackets) in the engine cylinder block and cylinder head and then out into the radiator. This helps to keep the engine from overheating; the hot coolant passes through the radiator where it cools down and then returns back to the engine. A water pump is usually driven by the engine through the drive belt. Sometimes it's driven by a timing belt. A water pump consists of the housing with the shaft rotating on the bearing pressed inside. At the outer side there is a pulley mounted on the shaft. At the inner side there is a seal to keep the coolant from leaking out and the impeller that acts like a centrifugal pump [6].

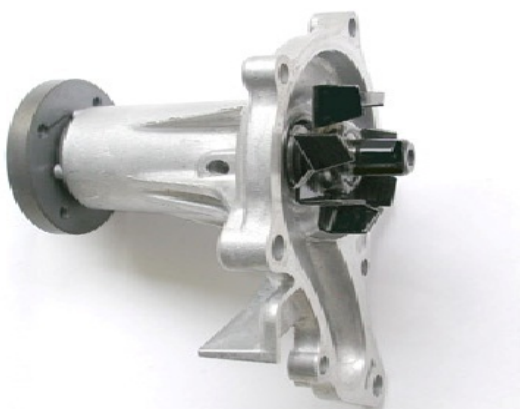

Figure 4: Water Pump

\section{ii. Alternator}

An automotive charging system is made up of three major components: the battery, the voltage regulator and an 
alternator. The alternator works with the battery to generate power for the electrical components of a vehicle, like the interior and exterior lights, and the instrument panel. An alternator gets its name from the term alternating current.

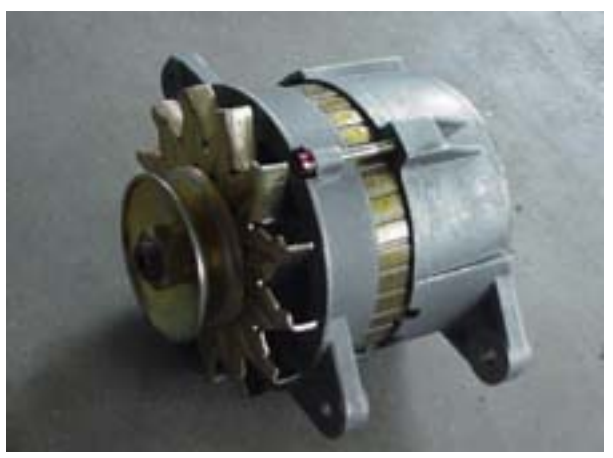

Figure 5: Alternator for Maruti 800

Alternators are typically found near the front of the engine and are driven by the crankshaft, which converts the pistons' up-and-down movement into circular movement. Some early model vehicles used a separate drive belt from the crankshaft pulley to the alternator pulley, but most cars today have a serpentine belt, or one belt that drives all components that rely on crankshaft power. Most alternators are mounted using brackets that bolt to a specific point on the engine. One of the brackets is usually a fixed point, while the other is adjustable to tighten the drive belt. Alternators produce AC power through electromagnetism formed through the stator and rotor relationship. The electricity is channeled into the battery, providing voltage to run the various electrical systems. [7]

The rotor and the stator are the two components that generate power. As the engine rotates the alternator pulley, the rotor spins past three stationary stator windings, or wire coils, surrounding a fixed iron core that makes up the stator. This is referred to as a three-phase current. The coil windings are evenly spaced at intervals of 120 degrees around the iron shaft. The alternating magnetic field from the rotor produces a subsequent alternating current in the stator. This AC current is fed through stator leads into a connecting set of diodes. Two diodes connect to each stator lead to regulate the current. The diodes are used to essentially block and direct the current. Since batteries need DC current, the diodes become a one-way valve that will only allow current to pass in the same direction.

Three-phase alternators have three sets of windings; they're more efficient than a single-phase alternator, which produce a single-phase AC current. When working properly, the three windings produce three currents that make up the three phases. Adding all three together produces the total AC output of the stator.

The two basic stator winding designs are delta wound and wye style. Delta wound are easily identifiable by their shape, as they're triangular. These windings allow for a high current flow at lower RPM. Wye windings resemble the flux capacitor seen in "Back to the Future." These windings are ideal for diesel engines, as they produce higher voltage than delta stators at even lower RPM.

After the AC/DC conversion, the resulting voltage is ready to use in the battery. Too much or too little voltage can damage the battery, as well as other electrical components. To ensure the correct amount, a voltage regulator determines when and how much voltage is needed in the battery. One of two types of regulators are found in most alternators: The grounded regulator works by controlling the amount of negative or battery ground going into the winding in the rotor, while a grounded field type works the other way around - by controlling the amount of battery positive. Neither poses an advantage over the other.

With so many components working to create the electricity vital for our vehicles, it's safe to say the alternator is a crucial component under the hood. But like many parts on our cars, they fail. The next section will give you an idea of how to determine if you are about to be stranded and what you can do if you need to replace your alternator.

\section{E. Consequence of Overheating}

If the engine overheats, the first thing that will happen is a gasoline engine will start to detonate. The engine will ping and start to lose power under load as the combination of heat and pressure exceed the octane rating of the fuel. If the detonation problem persists, the hammer-like blows may damage the rings, pistons or rod bearings.

Overheating can also cause pre-ignition. Hot spots develop inside the combustion chamber that becomes a source of ignition for the fuel. The erratic combustion can cause detonation as well as engine run-on in older vehicles with carburetors. Hot spots can also be very damaging and burn holes right through the top of pistons. Another consequence of overheating may be a blown head gasket. Heat makes aluminum swell almost three times faster than cast iron. [9]

The resulting stress can distort the head and make it swell in areas that are hottest like those between exhaust valves in adjoining cylinders, and areas that have restricted coolant flow like the narrow area that separates the cylinders. The typical aluminum head swells most in the middle, which can crush the head gasket if the head gets hot enough. This will cause a loss of torque in the gasket allowing coolant and combustion leaks to occur when the head cools.

\section{F. Engine Seizing}

Normally when an engine is "seized up" it is frozen to the point that the crankshaft will not turn in the bearings. This is caused by overheating. The parts that have seized or melted together could be main or rod bearings, piston rings

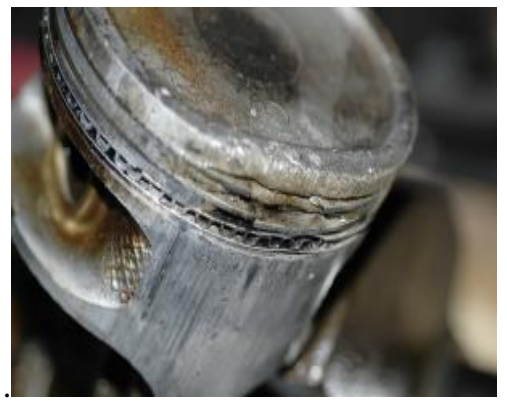

Figure 6: Piston after Engine Seizing 


\section{DetAILED DESCRIPTION OF THE PREFERRED EMBODIMENT}

The above mentioned consequences can be avoided by predetermination of slackness of the belt. The tightness can be indicated to the driver by the following proposal This apparatus consist of a simple potentiometer which can be used as a voltage divider or a potential divider to obtain a manually adjustability.it also consist of three pulley a belt, an external frame, alternator, circuit board, spring, roller etc.

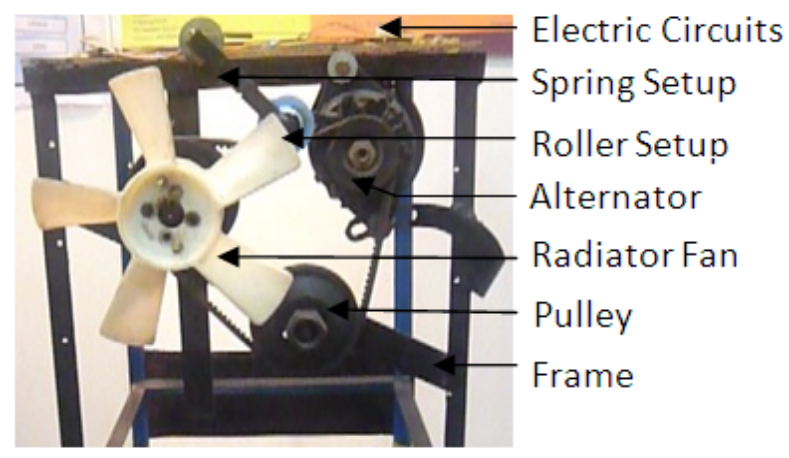

Figure 7: Apparatus Setup

Major components involved are potentiometer and LM3914, whose working is, discussed bellow

\section{A. Potentiometer}

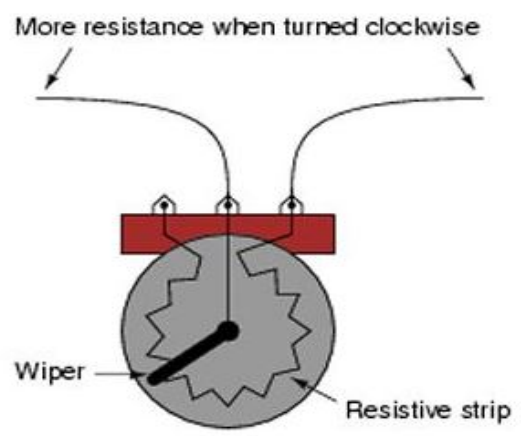

Figure 8: Potentiometer

In electronics, a voltage divider (also known as a potential divider) is a simple linear circuit that produces an output voltage (Vout) that is a fraction of its input voltage (Vin). Voltage division refers to the partitioning of a voltage among the components of the divider.

A simple example of a voltage divider consists of two resistors in series or a potentiometer. It is commonly used to create a reference voltage, and may also be used as a signal attenuator at low frequency

The formula governing a voltage divider is similar to that for a current divider, but the ratio describing voltage division places the selected impedance in the numerator, unlike current division where it is the unselected components that enter the numerator. Potentiometers work by having a resistive element inside. Both end terminals are attached to it, and do not move. The wiper travels along the strip when the knob is turned. It is commonly used to create a reference voltage, and may also be used as a signal attenuator at low frequency. The closer the wiper is to the end terminal it is wired in conjunction with, the less the resistance, because the path of the current will be shorter. The further away it moves from the terminal, the greater the resistance will be.

\section{B. Circuit Detail and Working of LM3914}

The LM3914 is a monolithic integrated circuit that senses analog voltage levels and drives LEDs, providing a linear analog display. A single pin changes the display from a moving dot to a bar graph. Current drive to the LEDs is regulated and programmable, eliminating the need for resistors. This feature is one that allows operation of the whole LM3914 system from less than $3 \mathrm{~V}$, there is a companion LM3915 device [10].

The LM3914 circuit contains its own adjustable reference and accurate voltage divider. [11] The low-bias-current input buffer accepts signals down to ground, or $\mathrm{V}$-, yet needs no protection against inputs of $35 \mathrm{~V}$ above or below ground. The buffer drives 10 individual comparators referenced to the precision divider. Indication non-linearity can thus be held typically to $1 / 2 \%$, even over a wide temperature range.

When all the circuit is connected properly then the apparatus denotes any deviations in the tightness of the belt in the system with the small LED display [12].

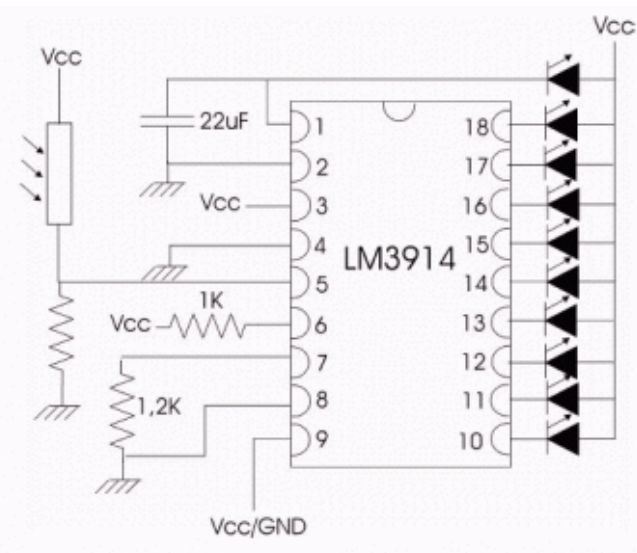

Figure 9: Circuit Diagram

\section{Working of the Apparatus}

The reference tightness is fixed initially in the system by placing the roller over the belt; if the belt gets loosened then the roller arrangement will get lowered. This lowering in the roller is converted in to the variation of resistance in potentiometer. [13]Since resistance is directly proportional to voltage, output voltage will be varied. This analog output is fed in to the LM3914 which provides a linear analog display by driving LED's.

\section{CONCLUSION}

The slackening of the serpentine belt or timing belts may lead to several problems as mentioned above which may even result in crisis to the driver. Our innovative proposal gives a feasible solution by using simpler and cost effective materials like potential divider and LM3914, etc. This acts as a preventive measure for the issue that has been discussed earlier. In future our proposal can be upgraded with the help of 
sensors and a micro controller to analyze and deal these slackening problems in more effective manner.

\section{REFERENCES}

[1] Chilton's Auto Repair Manual Pp. 5-24, 1993-1997

[2] Automotive technology: a systems approach: Vol. 2, Pp. 326, Jack Erjavec 1401, 2005

[3] Replacing Your Timing Belt - - Page 93-Popular Mechanics - Vol. 170, No. 6, June 1993.

[4] http://www.buzzle.com/articles/timing-belt-problems.html

[5] Total Automotive Technology -Pp. 87, Anthony E. Schwaller, Pp. 1080, 2004

[6] http://www.samarins.com/glossary/water_pump.html

[7] http://web.media.mit.edu/ nathan/nepal/ghatta/altantics.html

[8] http://web.media.mit.edu/ nathan/nepal/ghatta/alternator.html

[9] Chilton's Auto Repair Manual, 1993

[10] http://www.electronics-tutorials.com/devices/lm3914.htm

[11] http://www.energies.alba-annuaire.fr/data/lm/LM3914.pdf

[12] http://www.national.com/mpf/LM/LM3914.html\#Overview

[13] http://www.brighthub.com/engineering/electrical/articles/47625.aspx

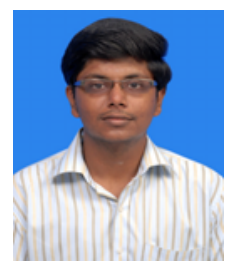

Sathian. C is a final year student of mechanical engineering from VelTech Engineering College, Anna University, Chennai, Tamilnadu. He has published a manuscript in CIIT International Journal of Automation and Autonomous Systems [ISSN 0974 - 9659] in Volume 3, No 3, in April 2011. He has attended many conferences and presented papers. He has also undergone summer industrial enrichment with A.T.I Hyderabad during June 2010.

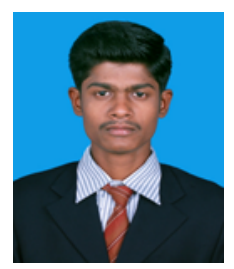

Kumar. B is a final year student of mechanical engineering from VelTech Engineering College, Anna University, Chennai, Tamilnadu, He is an active student member in SAEINDIA and has attended many conferences and presented papers. $\mathrm{He}$ has also undergone summer industrial enrichment with A.T.I Hyderabad in June 2010.

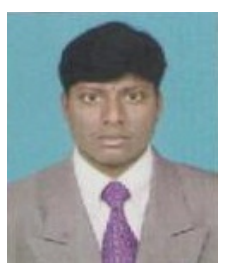

Raju. C is a final year student of mechanical engineering from VelTech Engineering College, Anna University, Chennai, Tamilnadu. He is an active student member in SAEINDIA. He has presented papers in various conferences and symposiums. 